\title{
Corneal Epithelial Defect in Adenoviral Conjunctivitis during Treatment with Corticosteroids
}

\section{Orucoglu $\mathrm{F}^{*}$ and Ayoglu I}

Bir Inci Eye Hospital, Istanbul, Turkey

\begin{abstract}
Adenovirus infection can involve many organ systems, including respiratory, ocular, metabolic, renal and gastrointestinal. Adenovirus infection is the principal pathogen in epidemics of conjunctivitis or keratoconjunctivitis. In this report, we present three cases of epithelial defect with adenoviral keratoconjunctivitis after initiation of topical corticosteroids.
\end{abstract}

Keywords: Adenoviral kerato conjunctivitis; Corneal defect; Corticosteroids

\section{Introduction}

Adenovirus can give rise to epidemic outbreaks and are characterized by a combination of health-care and community transmissions. Adenoviruses causes a wide range of illnesses, including respiratory infections, gastroenteritis, and keratoconjunctivitis [1]. Most adenoviral eye diseases clinically present one of the three classic syndromes: simple follicular conjunctivitis, pharyngoconjunctival fever, or Epidemic Kerato Conjunctivitis (EKC). Symptoms in the eye usually appear within 14 days after exposure and commonly include foreign body feeling, tearing, photophobia, and redness. The most frequent signs are predominantly conjunctival reddening, follicular reaction, conjunctival chemosis, petechiae and subconjunctival hemorrhages [2]. The appearance of pseudomembranes and corneal infiltrates are an additional late manifestations of EKC. Clinical illness typically lasts around 1-3 weeks and is usually self-limited. At this time, adenovirus keratoconjunctivitis treatment is focused on managing patient symptoms and avoiding the appearance of complications. The use of corticosteroids in the management of adenoviral conjunctivitis remains controversial and the often use of corticosteroids can lead to severe ocular complications as a result of uncontrolled virus proliferation.

We report three cases of epithelial defect with adenoviral keratoconjunctivitis after initiation of topical corticosteroids.

\section{Case Report}

\section{Case 1}

Five-year old boy presented on 22.06.2014 to our clinic due to increase of the symptoms on the left eye. Slit-lamp examination revealed eyelid swelling, conjunctival hyperemia and corneal defect on the left eye and conjunctival injection on the right. His eye disease started 6 days ago with tearing and redness on the left eye. A topical drop combination of Dexamethasone and tobramycin had been administered since then.

\section{Case 2}

Forty-five years old women presented with complaints of severe pain on the right eye on 22.06.2014. She was examined by an ophthalmologist 5 days ago with red eye and loteprednol and tobramycin combination has been started. On the day of presentation she had swollen eyelids, follicular reaction and epithelial defect in $4 \times 4 \mathrm{~mm}$ diameter on the right eye and follicular reaction and subconjunctival hemorrhages on the left (Figure 1a and 1b).

\section{Case 3}

Sixty-six years old male first presented with bilateral chemosis on 26.06.2014. Moxifloxacin and fluorometholone were initiated on day of first presentation. One day later, he returned with worsening of symptoms. Epithelial defect was revealed and artificial tears were added. The next day bandage contact lens was applied. He referred to our department for persistent epithelial defect on 10.07.2014. Slitlamp examination revealed large epithelial defect on the right eye and hyperemia on the left eye (Figure 2a and 2b).

All patients presented during same period of epidemic of adenovirus ocular disease in the area. The diagnosis was made on the basis of history and on the clinical findings of the patient. Infection first affected one eye then transmitted to the other in two patients. All patients were received topical corticosteroids from the beginning of the infection and they were still on corticosteroids during epithelial defect. The approach of the treatment were discontinuing topical corticosteroids, applying bandage contact lenses and continue with topical preservative free antibiotics and artificial tears. We observed dramatic improvement in 2 days and bandage contact lens was removed on day 3. All patients showed full epithelization and decrease of symptoms.

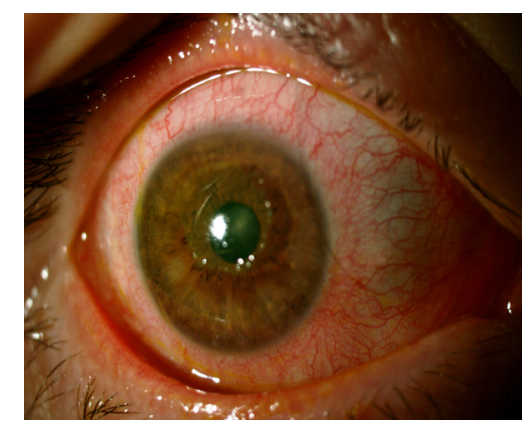

Figure 1a: Central epithelial defect on the right eye.

*Corresponding author: Orucoglu F, Bir Inci Eye Hospital, Istanbul, Turkey, Tel: 0850-302-00-22; E-mail: faikorucov@yahoo.co.uk

Received July 18, 2014; Accepted October 20, 2014; Published October 27, 2014

Citation: Orucoglu F, Ayoglu I (2014) Corneal Epithelial Defect in Adenoviral Conjunctivitis during Treatment with Corticosteroids. J Clin Case Rep 4: 429. doi:10.4172/2165-7920.1000429

Copyright: (c) 2014 Orucoglu F, et al. This is an open-access article distributed under the terms of the Creative Commons Attribution License, which permits unrestricted use, distribution, and reproduction in any medium, provided the original author and source are credited. 


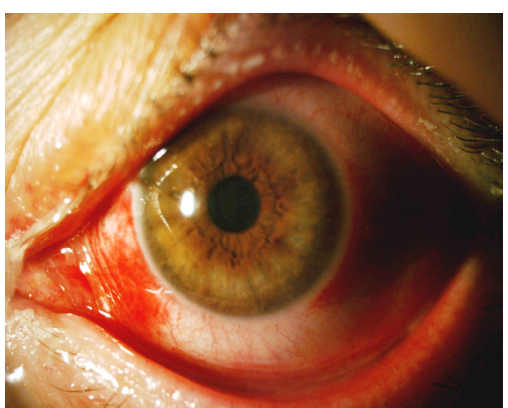

Figure 1b: Conjunctival hyperemia and hemorrhage on the left eye.

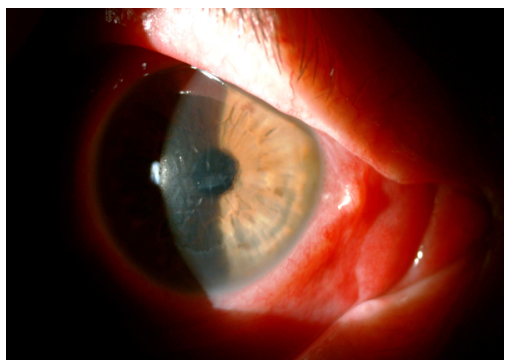

Figure 2a: Large epithelial defect on the left eye.

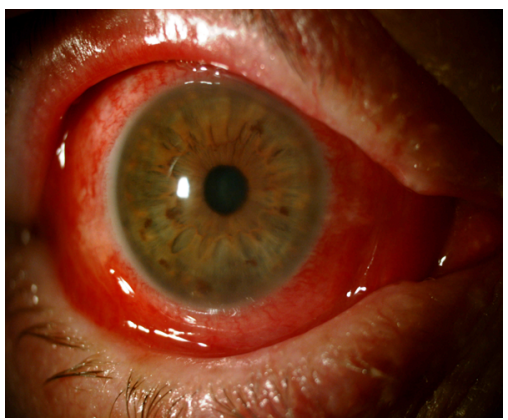

Figure 2b: Conjunctival hyperemia and hemorrhage on the right eye.

\section{Discussion}

Epidemic keratoconjunctivitis is an ocular surface infection associated with a marked inflammatory reaction, and symptoms of redness, irritation, tearing, blurry vision and sensitivity to light. The clinical diagnosis of an adenovirus infection is typically made based on the history and presenting signs and symptoms. Adenoviral keratoconjunctivitis is a self-limited disease and tends to resolve spontaneously within 1-3 weeks without significant complications [2]. To this date, there is no causative therapy or specific drug against the disease. Multiple treatments have been tried for this disease, but none of them seems to be completely effective. The treatment is focused mainly on managing the symptoms of the patient and includes palliative treatment, such as cool compresses, artificial tears and topical nonsteroidal anti-inflammatory drugs [3].

The often use of steroids in the eye is contraindicated for viral infections because they exacerbate the replication of virus and extend the duration of infection. Animal studies have demonstrated that the use of topical corticosteroids could increase the replication rate and prolongs adenovirus shedding $[4,5]$. It is shown that steroids, when combined with povidone-iodine may eradicate the secretion of virus and improve the manifestations of the disease [6].

In their early stages viral conjuncitivitis may be difficult to clinically differentiate from bacterial, herpetic, Chlamydia inclusion conjunctivitis or allergic conjunctivitis. This may lead to the misuse of medications including corticosteroids. The use of topical corticosteroids can lead to complications, and may mask serious conditions that require other interventions. The most common symptoms to be observed to make the difrentiation of adenoviral conjunctivitis are lid edema, serous discharge, diffuse hyperemia and chemosis, mixed papiller and follicular response of the conjunctiva, pseudomembrane formation in tarsal conjunctiva, subepithelial infiltrates and ipsilateral periauricular lymphadenopathy.

In all our patients who presented with corneal defect had been receiving topical corticosteroids. Corticosteroids were started in the beginning of the disease. All symptoms were decreased and epithelial defects showed improvement after discontinuing steroids. Wellknown complications of topical steroids include the formation of a geographic ulcer if given in the presence of a dendritic herpetic ulcer [7]. There may be a similar mechanism of developing epithelial defect in adenoviral keratoconjunctivitis.

The use of topical corticosteroids should be avoided in the early stages of adenoviral keratoconjunctivitis. As steroids may exacerbate symptoms and may lead to epithelial problems.

Consequently, the use of topical corticosteroids should be restricted to complicated cases with pseudomembranes or subepithelial infiltrates where visual acuity is significantly impaired. In case of early initiation of steroids, it should be combined with povidone-iodine.

\section{References}

1. Yamadera S, Yamashita K, Akatsuka M, Kato N, Hashido M, et al. (1995) Adenovirus surveillance, 1982-1993, Japan. A report of the National Epidemiological Surveillance of Infectious Agents in Japan. Jpn J Med Sci Biol 48: $199-210$

2. Ghebremedhin B (2014) Human adenovirus: Viral pathogen with increasing importance. Eur J Microbiol Immunol (Bp) 4: 26-33.

3. Gordon YJ, Araullo-Cruz T, Romanowski EG (1998) The effects of topical nonsteroidal anti-inflammatory drugs on adenoviral replication. Arch Ophthalmol 116: 900-905.

4. Romanowski EG, Yates KA, Gordon YJ (2001) Short-term treatment With potent topical corticosteroid of an acute ocular adenoviral infection in the New Zealand white rabbit. Cornea 20: 657-660.

5. Vajpayee RB, Sharma N, Chand M, Tabin GC, Vajpayee M, et al. (1998) Corneal superinfection in acute hemorrhagic conjunctivitis. Cornea 17: 614617.

6. Clement C, Capriotti JA, Kumar M, Hobden JA, Foster TP, et al. (2011) Clinica and antiviral efficacy of an ophthalmic formulation of dexamethasone povidoneiodine in a rabbit model of adenoviral keratoconjunctivitis. Invest Ophthalmo Vis Sci 52: 339-344.

7. Carter CA, Easty DL, Walker SR (1981) Experimental ulcerative herpetic keratitis. II. Influence of topical corticosteroid in immunised rabbits. $\mathrm{Br} J$ Ophthalmol 65: 388-391. 